\title{
Anterior dislocation of the shoulder with ipsilateral humerus shaft fracture
}

\author{
Pradhan I ${ }^{1}$, Banskota AK ${ }^{2}$ \\ ${ }^{1}$ Orthopaedic Surgeon, ${ }^{2}$ Professor, Department of Orthopaedic Surgery, B\&B Hospital, Kathmandu University Teaching \\ Hospital, Gwarko, Lalitpur, Nepal
}

\begin{abstract}
Anterior dislocation of the shoulder joint with ipsilateral humerus shaft fracture is a very rare injury. Until July 2006 only 18 cases have been reported in the literature. Here we report a case of Anterior Shoulder dislocation with greater tuberosity and shaft of humerus fracture. The patient was treated by Open reduction and internal fixation of the shaft fracture followed by closed reduction of the dislocation. A post-operative rehabilitation program was instituted for complete rehabilitation of the shoulder function.
\end{abstract}

Key words: Shoulder dislocation, Ipsilateral, Humerus, Fracture, Open reduction.

\begin{abstract}
Choulder dislocation with ipsilateral humerus fracture usually occurs as a result of a major trauma and is an uncommon but serious injury. In the English literature till July 2006 only 18 cases have been reported. Here we report a case with Shoulder dislocation with greater tuberosity fracture and ipsilateral shaft fracture, its mechanism and outcome of management.
\end{abstract}

\section{Case report}

A 35 year old male patient presented to our Emergency department three hours after a road traffic accident. He had sustained a fall from his speeding motor bike and landed on the right side of the body with his right upper extremity trapped beneath. On arrival at Emergency department, he was conscious, alert and his right arm was swollen and deformed. There were no open wounds and there were no distal Neurovascular (NV) deficit. There were no other injuries. Radiographic evaluation showed a fracture in the mid shaft of the right humerus and a dislocation of the right shoulder joint with displaced fracture of the greater tuberosity.

A closed reduction of the shoulder joint was not attempted as it was thought not possible to achieve traction in the arm to reduce the shoulder. So surgery was planned to internally fix the shaft fracture and then to provide traction to reduce the shoulder joint. An open reduction and internal fixation of the shaft fracture was done through anterolateral Deltopectoral approach using eight hole $4.5 \mathrm{~mm}$ Dynamic Compression Plate (DCP) with six proximal and six distal cortical screw fixation. Closed reduction was successful in reducing the dislocated shoulder joint but the reduction was unstable on minimal traction. The displaced greater tuberosity was also internally fixed with $6.5 \mathrm{~mm}$ cancellous screw with a washer. Post-operative x-ray was done to confirm reduction of the shoulder and the shoulder joint was immobilized by bandaging the arm to the trunk with round the trunk body bandaging. The post-operative recovery of the patient was uneventful and the patient was discharged on the fifth post-operative day with a velpaeu bandage. At three weeks, pendulum exercises of the shoulder were instituted and at six weeks, passive as well as active shoulder exercises were allowed. Fracture union was achieved at 12 weeks and full function of the shoulder was achieved at six months follow up. 


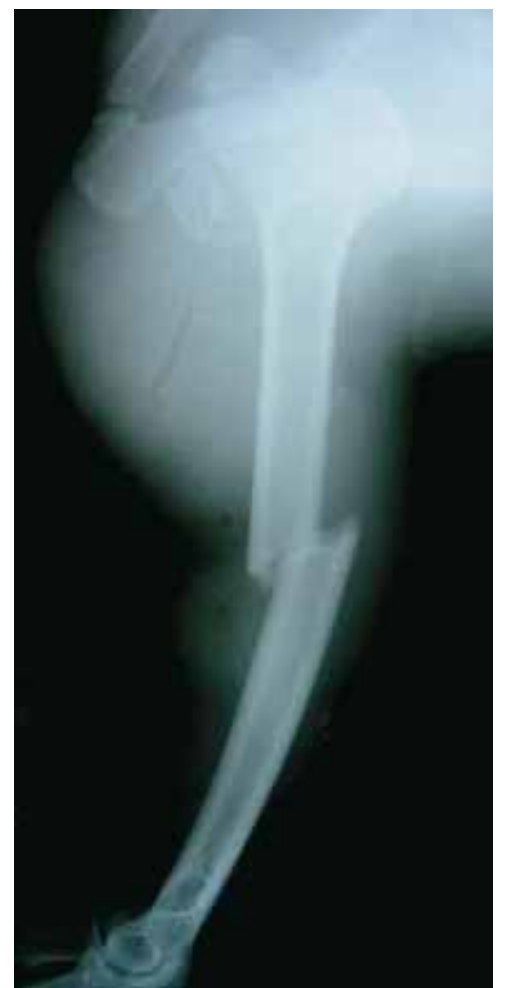

Fig 1: Radiograph showing anterior dislocation of shoulder with fracture shaft of humerus and displaced greater tuberosity fracture

\section{Discussion}

Dislocation of the glenohumeral joint with ipsilateral humerus shaft fracture is a very rare injury reported first in the literature by Winderman et al in $1940^{1}$. Our case not only had fracture of the shaft associated with the shoulder dislocation but also a displaced greater tuberosity fracture. This may well be the first reported case of shoulder dislocation with shaft and greater tuberosity fracture of the ipsilateral humerus.

There have been so far 18 similar cases reported in the world literature ${ }^{2,3}$.

Two mechanisms for such injuries have been proposed by previous authors. Sankaran-Kutty suggested that force exerted along the axis of the humerus simultaneously fractured the humerus and dislocated the shoulder ${ }^{4}$. Whereas Kontakis proposed that the shoulder dislocated first and subsequent bending or torsion forces fractured the shaft of the humerus ${ }^{5}$. Our patient was involved in a high velocity accident where the extremity was entrapped by the body weight, so the mechanism of injury might have been simultaneous fracture and dislocation.

Various methods of treatment have been described but no consensus in the best method of treatment for this

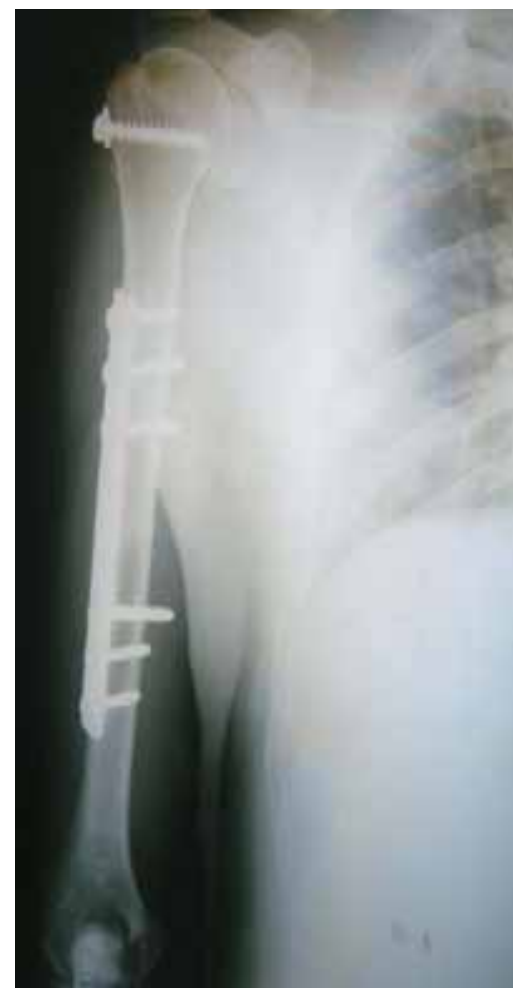

Fig 2: Radiograph showing fracture healing at 3 months post-operative

kind of combined injury has been proposed. Due to the nature of the injury it has been treated on an emergency basis. Shoulder dislocation has been reported to have been successfully reduced closed under general anesthesia, whereas failure of closed reduction under emergency environment has also been reported. But open reduction of the dislocation is seldom necessary. The fracture of the shaft has been fixed by various means such as external fixators, intramedullary nails and dynamic compression plates with consistent results in fracture union.

We treated our patient with open reduction and internal fixation of the fracture with DCP, followed by closed reduction of the shoulder joint which was spontaneous with minimal traction. But the reduction was felt to be unstable and a more conservative approach on postoperative rehabilitation was undertaken. Patient was immobilized in a Velpaeu banadage for three weeks when shoulder pendulum exercises were started. Passive and active range of motion (ROM) of the shoulder was started at six weeks. Fracture union was achieved at 12 weeks and full ROM of the shoulder gained by six months. 


\section{Conclusion}

Fracture dislocations around the glenohumeral joint are not uncommon but a diaphyseal fracture of the humerus with an anterior shoulder dislocation is a very rare injury. With prompt treatment and a diligent rehabilitation protocol, near normal function was restored. Even though the dislocation was noted to be unstable, the association of a greater tuberosity fracture with an anterior dislocation is known to be associated with significantly reduced risks of redislocation.

\section{References}

1. Winderman Dislocation of the shoulder with fracture of the shaft of the humerus. Bull Hospt Jt Dis Orthop Inst 1940;1:23-5.

2. Chen CH, Lai PL, Chen WJ, Shih CH. Simultaneous anterior dislocation of the shoulder and fracture of the ipsilateral humerus shaft. International Orthopaedics 1998;22:65-7.
3. Sasahige Y, Kurata T, Masuda Y, Shimono K, Nagata Y. Dislocation of the shoulder joint with ipsilateral humerus shaft fracture: two case reports. Acta Orthop Trauma Surg. 2006;126:252-7.

4. Sankaran-Kutty M. Sadat-Ali M. Dislocation of the shoulder and ipsilateral shaft fracture. Acta Orthop Trauma Surg. 1989;108:60-2.

5. Kontakis GM, Galankis IA, Steripoulous KA. Dislocation of the shoulder and ipsilateral fracture of the humeral shaft: case reports and literature review. J Trauma 1995;39:990-2. 